\title{
PROPRIEDADES REDOX E GRUPOS FUNCIONAIS DE ÁCIDOS HÚMICOS ISOLADOS DE ADUBOS ORGÂNICOS ${ }^{(1)}$
}

\author{
Marihus Altoé Baldotto ${ }^{(2)}$, Luciano Pasqualoto Canellas ${ }^{(3)}$, Maria \\ Cristina Canela ${ }^{(4)}$, Marcelo Luiz Simões ${ }^{(5)}$, Ladislau Martin-Neto ${ }^{(5)}$, \\ Maurício Paulo Ferreira Fontes ${ }^{(6)}$ \& Ary Carlos Xavier Velloso ${ }^{(3)}$
}

\begin{abstract}
RESUMO
Dependendo das condições do sistema, os ácidos húmicos podem atuar como oxidantes ou redutores. Nos sistemas naturais, o fluxo de elétrons está diretamente associado à quantidade e à qualidade do húmus. O potencial do eletrodo e a capacidade de oxidação informam sobre os fatores intensidade e capacidade dos sistemas redox, respectivamente. Estudos prévios têm aventado a hipótese de que radicais livres nos ácidos húmicos participam dessas reações redox. No presente estudo, seis ácidos húmicos isolados de adubos orgânicos foram titulados com um oxidante $\left(\mathrm{I}_{2}\right)$ em atmosfera inerte e condições especificadas. Os ácidos húmicos apresentaram valores do potencial formal-padrão do eletrodo semelhantes: entre 0,773 e $0,794 \mathrm{~V}$ a $25^{\circ} \mathrm{C}$. A capacidade de oxidação dos ácidos húmicos variou de 3,88 a 4,39 $\mathrm{mol}_{\mathrm{c}} \mathrm{kg}^{-1}$ a pH 5,0 e de 5,35 a 7,89 $\mathrm{mol}_{\mathrm{c}} \mathrm{kg}^{-1}$ a pH 7,0. Foi observada correlação positiva e significativa entre a capacidade de oxidação dos ácidos húmicos e as suas concentrações de grupos funcionais fenólicos, quinonas e semiquinonas.
\end{abstract}

Termos de indexação: química do solo, substâncias húmicas, potencial redox, capacidade de oxidação, humificação.

\footnotetext{
(1) Parte da Tese de Doutorado do primeiro autor apresentada à Universidade Estadual do Norte Fluminense Darcy Ribeiro (UENF). Trabalho financiado pelo CNPq e pela FAPERJ. Recebido para publicação em setembro de 2006 e aprovado em fevereiro de 2007.

(2) Pesquisador do Laboratório de Solos (LSol) do Centro de Ciências e Tecnologias Agropecuárias (CCTA) da UENF. Av. Alberto Lamego 2000, CEP 28013-602 Campos dos Goytacazes (RJ). Bolsista Pós-DOC da FAPERJ. E-mail: marihus@uenf.br

(3) Professor do LSol do CCTA da UENF. Av. Alberto Lamego 2000, CEP 28013-602 Campos dos Goytacazes (RJ). Bolsista do CNPq. E-mails: canellas@uenf.br; velloso@uenf.br

(4) Professora do Laboratório de Ciências Químicas (LCQui) do Centro de Ciências e Tecnologias (CCT) da UENF. Av. Alberto Lamego 2000, CEP 28013-602 Campos dos Goytacazes (RJ). Bolsista do CNPq. E-mail: mccanela@uenf.br

(5) Pesquisador da Embrapa Instrumentação Agropecuária, Caixa Postal 741, CEP 13560-970 São Carlos (SP). Bolsista do CNPq. E-mails: marcelo@cnpdia.embrapa.br; martin@cnpdia.embrapa.br

(6) Professor do Departamento de Solos da Universidade Federal de Viçosa - UFV. Av. PH Rolfs s/n, CEP 36571-000 Viçosa (MG). Bolsista do CNPq. E-mail: mpfontes@ufv.br
} 


\title{
SUMMARY: REDOX PROPERTIES AND FUNCTIONAL GROUPS OF HUMIC ACIDS ISOLATED FROM ORGANIC RESIDUES
}

\begin{abstract}
According to the system conditions, humic acids act as oxidants or as reductants. In natural systems the electron flow is directly associated with humus content and quality. Electrode potential and oxidation capacity inform about the intensity and capacity of the redox systems. In previous studies it has been assumed that free radicals participate in humic acid redox reactions. In our study six humic acids isolated from composed organic residues were titrated with an oxidant $\left(I_{2}\right)$ in inert atmosphere and specified conditions. Humic acids presented similar standard formal electrode potentials, with values between 0.773 and $0.794 \mathrm{~V}$, at $25^{\circ} \mathrm{C}$. The oxidation capacity of humic acids varied from 3.88 to $4.39 \mathrm{~mol}_{c} \mathrm{~kg}^{-1}$ at pH 5.0 and from 5.35 to $7.89 \mathrm{~mol}_{\mathrm{c}} \mathrm{kg}^{-1}$ at $\mathrm{pH}$ 7.0. Positive and significant correlations were observed between the oxidation capacity and the concentrations of the phenolic, quinone and semiquinone functional groups in the humic acids.
\end{abstract}

Index terms: soil chemistry, humic substances, redox potential, oxidation capacity, humification.

\section{INTRODUÇÃO}

$\mathrm{O}$ aumento e a estabilidade dos estoques de $\mathrm{C}$ no sistema solo reduzem a emissão de $\mathrm{CO}_{2}$ para a atmosfera e o agravamento do efeito estufa, contribuindo com estratégias de desenvolvimento limpo. No solo, a estabilidade química do $\mathrm{C}$ ocorre com a formação de substâncias húmicas (SH), por meio de um processo genericamente denominado humificação. Esse processo pode ser estimado pelo aumento da concentração de radicais livres do tipo semiquinonas (CRLS) nos ácidos húmicos (AH), determinados por meio da ressonância paramagnética eletrônica (RPE) (Martin Neto et al. 1994a, b; Pérez et al., 2004). Com o avanço da humificação ocorre a policondensação e a conjugação de estruturas insaturadas nos AH (Piccolo, 2001). Grupamentos fenólicos formados durante o processo de decomposição da matéria orgânica são convertidos em quinonas, via reações de oxidação, as quais são precursoras de radicais livres do tipo semiquinonas nos $\mathrm{AH}$ (Milori et al., 2002; Budziak et al., 2004; Rivero et al., 2004), que se estabilizam com o avanço da humificação.

Os radicais livres do tipo semiquinonas dos $\mathrm{AH}$ são grupos doadores de elétrons e, por meio de titulações redox iodimétricas, podem ser oxidados pelo iodo $\left(\mathrm{I}_{2}\right)$. Dessa forma, tais titulações permitiriam, analogamente à RPE, estimar o grau de humificação dos AH. No entanto, nem toda a atividade redox dos AH pode ser atribuída a CRLS, sendo necessárias informações adicionais sobre a relação entre os diferentes grupos funcionais e o poder redutor dos $\mathrm{AH}$ (Helburn \& MacCarthy, 1994; Matthiessen, 1995; Lovley et al., 1998; Scott et al., 1998; Struyk \& Sposito, 2001).

Scott et al. (1998) e Lovley et al. (1996, 1998) estudaram a atividade redox dos $\mathrm{AH}$ e indicaram que os grupos quinonas são os principais responsáveis pela transferência de elétrons entre microrganismos, $\mathrm{AH}$ e espécies sólidas de Fe(III). Segundo o mecanismo indicado pelos autores, os radicais semiquinonas po- dem originar-se quando grupos quinonas presentes nos AH são reduzidos, permanecendo estáveis no ambiente. Os grupos semiquinonas podem ser reduzidos a radicais hidroquinonas, ainda mais estáveis. Alternativamente, grupos fenólicos contidos nos AH também podem sofrer oxidação e formar radicais semiquinonas, que, se oxidados, podem resultar em benzoquinonas (Struyk \& Sposito, 2001). Adicionalmente, o segundo mecanismo citado indica que parte da quantidade de elétrons transferíveis pelos $\mathrm{AH}$ é proveniente da oxidação do $\mathrm{Fe}(\mathrm{II})$ ligado a grupos fenólicos. Na reação, um agente oxidante, como o $\mathrm{I}_{2}$, recebe um elétron a partir do complexo AH-Fe(II), reduzindo-o a um grupo semiquinona (Struyk \& Sposito, 2001). Dessa forma, a concentração de radicais livres de tipo semiquinona pode ser mantida pela "intra-oxidação" durante a titulação redox dos AH (Struyk \& Sposito, 2001). Outros estudos verificaram a ocorrência da ligação entre o Fe(III) e moléculas orgânicas (Schwarzenbach et al., 1990; Martin-Neto et al., 1994 b), entre moléculas poluentes, como os compostos nitroaromáticos e metanos polialogenados, sendo o Fe(III) reduzido a Fe(II). Os autores sugerem que um ciclo similar seja esperado entre a matéria orgânica humificada e espécies de Fe.

A metilação dos $\mathrm{AH}$ com diazometano $\left(\mathrm{CH}_{2} \mathrm{~N}_{2}\right)$ converte grupos ácidos em $\mathrm{R}-\mathrm{OCH}_{3}$, que, no caso de $\mathrm{SH}$, são denominados humatos de metila. Especificamente, grupos fenólicos e quinonas dos AH podem ser acetilados com anidrido acético em piridina, formando ésteres (Stevenson, 1994; Sachs et al., 2002). A titulação redox desses derivados metilados e acetilados permite verificar a participação dos grupos ácidos e dos resíduos de quinonas nas reações redox envolvendo AH.

As titulações redox de $\mathrm{AH}$ permitem estimar o potencial formal-padrão do eletrodo $\left(\mathrm{F}_{\mathrm{H}}{ }^{\circ}\right)$ e a capacidade de oxidação (COx), os fatores intensidade e capacidade da atividade de elétrons, respectivamente, análogos ao $\mathrm{pH}$ e a capacidade de neutralização para o caso de prótons (Sposito, 1989). $\mathrm{O}^{\mathrm{F}} \mathrm{E}_{\mathrm{H}}{ }^{\circ}$ pode ser medido 
utilizando-se um eletrodo combinado, constituído de um eletrodo sensível em contato com um eletrodo de referência. O eletrodo sensível responde à transferência de elétrons na semi-reação redox da espécie da qual se deseja medir o potencial, e o eletrodo de referência mantém seu potencial constante, gerando uma força eletromotriz (f.e.m.), que é resultante da diferença de potencial eletroquímico entre os dois eletrodos (Stumm \& Morgan, 1996). O valor do ${ }^{\mathrm{F}} \mathrm{E}_{\mathrm{H}}{ }^{\mathrm{o}}$ possibilita a determinação das atividades de oxidantes e redutores para um determinado par redox. A COx de uma substância pode ser utilizada na determinação da quantidade de elétrons transferíveis nas reações redox e é definida como o número de mols de carga por unidade de massa que um composto é capaz de transferir a um forte oxidante $\left(\mathrm{mol}_{\mathrm{c}} \mathrm{kg}^{-1}\right)$.

Este estudo teve como objetivos determinar, por meio de titulações redox iodimétricas, a capacidade de $\mathrm{AH}$ isolados de adubos orgânicos, e de seus derivados químicos acetilados e metilados, transferirem elétrons e relacioná-la com a concentração de grupos funcionais carboxílicos, fenólicos, quinonas e semiquinonas presentes em sua estrutura.

\section{MATERIAL E MÉTODOS}

\section{Amostras de ácidos húmicos}

Para isolamento dos AH, seis adubos orgânicos foram escolhidos: (a) vermicomposto; (b) composto de esterco de curral; (c) composto de esterco de aviário; (d) composto da mistura em partes iguais, de esterco de curral e de aviário; (e) composto de torta de filtro e (f) composto de resíduo orgânico de lixo domiciliar urbano.

O vermicomposto foi preparado a partir de esterco de curral e de minhocas vermelhas da Califórnia (Eisenia foetida). A coleta do material foi realizada 90 dias após a instalação das pilhas de esterco, o que corresponde a 45 dias depois de colocadas as minhocas. Os estercos de curral e de aviário foram coletados na "Fazendinha Agroecológica" da Embrapa Agrobiologia, Seropédica-RJ. A torta de filtro e o lixo urbano foram amostrados, respectivamente, na Usina Paraíso, localizada no município de Campos dos GoytacazesRJ e na Companhia de Limpeza Urbana (Comlurb) do município do Rio de Janeiro. A compostagem da matéria orgânica foi realizada conforme os procedimentos usuais adotados na "Fazendinha Agroecológica", que envolvem, principalmente, o controle diário da temperatura e o revolvimento semanal das pilhas durante a fase termófila.

Os AH foram isolados dos adubos orgânicos conforme as recomendações da Sociedade Internacional de Substâncias Húmicas (IHSS, 2006), usando-se $\mathrm{NaOH}$ 0,1 mol L-1 sob atmosfera de $\mathrm{N}_{2}$. Após agitação por $24 \mathrm{~h}$, o material foi centrifugado a $5.000 \mathrm{~g}$ por $30 \mathrm{~min}$. O sobrenadante foi coletado e o $\mathrm{pH}$ do extrato foi imediatamente ajustado a 1,5 com $\mathrm{HCl} 6 \mathrm{~mol} \mathrm{~L}^{-1}$. Depois de 18 h, a fração ácidos fúlvicos foi sifonada e descartada. O material remanescente (precipitado de AH) foi solubilizado em $\mathrm{NaOH} 0,1 \mathrm{~mol} \mathrm{~L}^{-1}$ e centrifugado a $5.000 \mathrm{~g}$ por $10 \mathrm{~min}$, e o sobrenadante, descartado. A dissolução e a reprecipitação dos AH foi repetida por mais duas vezes. A seguir, o precipitado de $\mathrm{AH}$ foi solubilizado e permaneceu em $\mathrm{HF}+\mathrm{HCl} 5 \%$ por $48 \mathrm{~h}$, visando à remoção de resíduos de minerais de argila e de íons paramagnéticos, sendo a seguir centrifugado a $5.000 \mathrm{~g}$. Os AH foram lavados com $200 \mathrm{~mL}$ de $\mathrm{HCl} 0,01 \mathrm{~mol} \mathrm{~L}^{-1}$, centrifugados a $5.000 \mathrm{~g}$. A seguir, o precipitado de $\mathrm{AH}$ foi lavado com água destilada até teste negativo para $\mathrm{Cl}^{-}$, usando-se $\mathrm{AgNO}_{3} 0,1 \mathrm{~mol} \mathrm{~L}^{-1}$, e depois transferido para membranas de diálise de $10 \mathrm{~mL}$ (cut-off 14 KDa, Thomas Sci.). Após a diálise, até a obtenção de condutividade elétrica igual à da $\mathrm{H}_{2} \mathrm{O}$ destilada $\left(1,0 \mu \mathrm{S} \mathrm{cm}^{-1}\right)$, os $\mathrm{AH}$ foram liofilizados e armazenados em dessecador.

\section{Derivados acetilados e metilados}

Os $\mathrm{AH}$ isolados foram submetidos à derivação química por metilação e acetilação (Sachs et al., 2002). Os derivados acetilados foram obtidos após a reação com anidrido acético em presença de piridina, sob agitação magnética contínua à temperatura ambiente, durante uma semana. $\mathrm{O}$ processo de metilação de $\mathrm{AH}$ foi conduzido com diazometano $\left(\mathrm{CH}_{2} \mathrm{~N}_{2}\right)$, produzido a partir do reagente $\mathrm{N}$-metil- $\mathrm{N}$-nitrosotolueno- $p$ sulfonamida em éter etílico, na presença de metanol. $\mathrm{O}$ procedimento de metilação dos $\mathrm{AH}$ foi repetido por três vezes e finalizado quando a incorporação do diazometano aos $\mathrm{AH}$ foi completada, indicada pela coloração amarela do $\mathrm{CH}_{2} \mathrm{~N}_{2}$.

As reações de metilação e acetilação dos AH foram monitoradas por meio da espectroscopia na região do infravermelho com transformada de Fourier (IV-TF), entre as faixas de 400 e 4.000 número de ondas $\mathrm{cm}^{-1}$. Os espectros foram obtidos usando-se pastilhas com $1 \mathrm{mg}$ de $\mathrm{AH}$ em $100 \mathrm{mg}$ de $\mathrm{KBr}$, em um aparelho Shimadzu 83000 (Stevenson, 1994).

\section{Titulações redox}

As titulações redox consistiram da oxidação dos $\mathrm{AH}$ estudados com $\mathrm{I}_{2}$ (iodimetria), sob atmosfera inerte (Struyk \& Sposito, 2001). Utilizou-se um frasco hermético (Figura 1), mantendo-se a temperatura do titulado a $25^{\circ} \mathrm{C}$ por meio de fluxo de água contínuo, proporcionado pelo acoplamento do frasco de titulação a um equipamento de "banho termostatizado" (Microquímica ${ }^{\circledR}$ Banho MQBCT 99-20).

Preparou-se a tampa do frasco para acomodar um eletrodo combinado para $\mathrm{pH}$ (peagâmetro Quimis Q400A) e um eletrodo redox combinado (Analyser 6A05-GK AgCl, $\mathrm{Ag}|| \mathrm{Pt}$ ), além de um tubo para entrada de gás (Ar) e uma microbureta de $2 \mathrm{~mL}$ (Gilmont $^{\circledR}$ GS-1200-A, com subdivisões de $2 \mu \mathrm{L}$ ), usando-se silicone para vedação. 


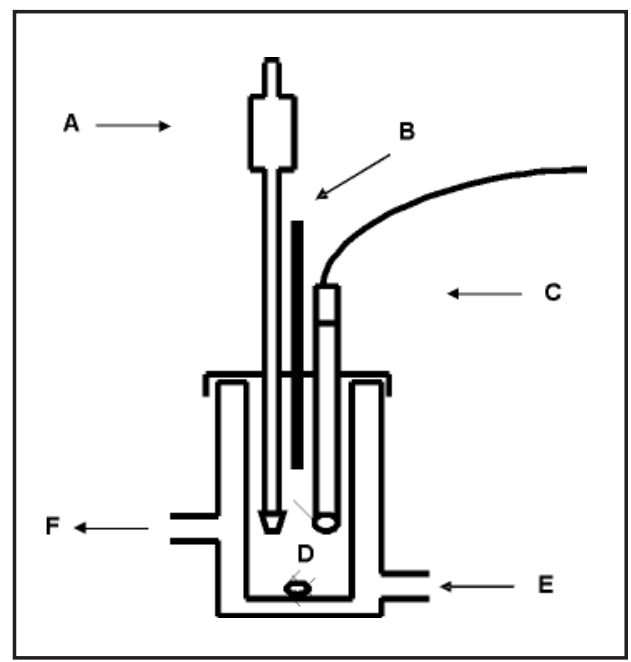

Figura 1. Esquema do equipamento de titulação redox. A: microbureta; B: tubo para entrada de Ar; C: eletrodo de Pt combinado (referência AgCl, Ag); D: agitador magnético; E e F: entrada e saída de água a $25^{\circ} \mathrm{C}$, respectivamente.

No preparo do titulado, adicionaram-se ao frasco de titulação $125 \mathrm{~mL}$ de solução-tampão fosfato $\left(\mathrm{Na}_{2} \mathrm{HPO}_{4} 0,05 \mathrm{~mol} \mathrm{~L}^{-1} \mathrm{e} \mathrm{NaH}{ }_{2} \mathrm{PO}_{4} \cdot \mathrm{H}_{2} \mathrm{O} 0,05 \mathrm{~mol} \mathrm{~L}^{-1}\right)$

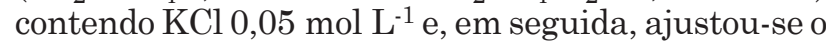
$\mathrm{pH}$ com $\mathrm{HCl}$ ou $\mathrm{NaOH}$ para $\mathrm{pH} 5,00$ ou 7,00 .

A solução foi agitada magneticamente e borbulhada com Ar por $60 \mathrm{~min}$, para depleção de $\mathrm{O}_{2}$. Acrescentouse $\mathrm{AH}$ à solução para obter uma concentração em suspensão de $50 \mathrm{mg} \mathrm{L}^{-1}(6,25 \mathrm{mg}$ AH suspensos em $125 \mathrm{~mL}$ de solução-tampão fosfato $0,05 \mathrm{~mol} \mathrm{~L}^{-1} \mathrm{com}$ $\mathrm{KCl}$ 0,05 mol L-1). Novamente, agitou-se e borbulhouse a suspensão com Ar durante $30 \mathrm{~min}$. Em seguida, ajustou-se o $\mathrm{pH}$ da suspensão para 5 ou $7 \mathrm{com} \mathrm{HCl}$ ou $\mathrm{NaOH}$, para início da titulação.

Para cada titulação, calibrou-se o peagâmetro utilizando soluções-tampão a pH 4 e a pH 7 . O eletrodo redox foi calibrado com solução-tampão redox férricoferrosa, preparada com sulfato ferroso amoniacal $0,100 \mathrm{~mol} \mathrm{~L}^{-1}$, sulfato férrico amoniacal 0,100 $\mathrm{mol} \mathrm{L}^{-1}$ e $\mathrm{H}_{2} \mathrm{SO}_{4} 1,00 \mathrm{~mol} \mathrm{~L}^{-1}$. A leitura da f.e.m. cela em $439 \pm 2 \mathrm{mV}$ indicou o correto funcionamento do eletrodo (Light, 1972).

Padronizou-se a solução de $\mathrm{I}_{2}$, para cada titulação, utilizando tiossulfato de sódio, de acordo com as reações propostas por Bard et al. (1985):

$$
\begin{array}{ll}
2 \mathrm{~S}_{2} \mathrm{O}_{3}{ }^{2-}(\mathrm{aq})=\mathrm{S}_{4} \mathrm{O}_{6}{ }^{2-}(\mathrm{aq})+2 \mathrm{e}^{-} & \mathrm{E}_{\mathrm{H}}{ }^{\mathrm{o}}=-0,080 \mathrm{~V} \\
\mathrm{I}_{2}(\mathrm{aq})+2 \mathrm{e}^{-}=2 \mathrm{I}^{-}(\mathrm{aq}) & \mathrm{E}_{\mathrm{H}}^{\mathrm{o}}=+0,621 \mathrm{~V} \\
\hline 2 \mathrm{~S}_{2} \mathrm{O}_{3}{ }^{2-}(\mathrm{aq})+\mathrm{I}_{2}(\mathrm{aq})=\mathrm{S}_{4} \mathrm{O}_{6}{ }^{2-}(\mathrm{aq})+2 \mathrm{I}^{-}(\mathrm{aq}) & \overline{\mathrm{E}_{\mathrm{H}}{ }^{o}=+0,541 \mathrm{~V}}
\end{array}
$$

Nas titulações utilizaram-se incrementos de $2 \mu \mathrm{L}$ de solução $0,025 \mathrm{~mol} \mathrm{~kg}^{-1} \mathrm{de}_{2}\left(\mathrm{~mol} \mathrm{~kg}{ }^{-1}=\right.$ molinidade, ou seja, o número de mols de $\mathrm{I}_{2}$ por $\mathrm{kg}$ de solução) com densidade conhecida e, após cada incremento de titulante, a solução foi agitada e borbulhada com Ar por mais 30 segundos, para facilitar a homogeneização do sistema. Em seguida, registrou-se a leitura da f.e.m.cela após cada adição de titulante, sempre quando a variação no aparelho foi menor ou igual a $2,0 \mathrm{mV} \mathrm{\text {min}^{-1 }}$. O ponto final da titulação redox de $\mathrm{AH}$ foi atingido quando valores constantes de f.e.m.cela foram registrados após a adição de oxidante.

As curvas de titulação redox foram obtidas relacionando-se num gráfico os valores de f.e.m.cela (eixo das ordenadas) de acordo com a $\mathrm{COx}$ dos $\mathrm{AH}$ (eixo das abscissas). O valor da $\mathrm{COx}$ foi determinado pelo número de mols de $\mathrm{I}_{2}$ (aq) reduzidos a $\mathrm{I}^{-}{ }_{(\mathrm{aq})}$ na reação por unidade de massa de $\mathrm{AH}\left(\mathrm{mol}_{\mathrm{c}} \mathrm{kg}^{-1}\right)$, usando-se a equação (Struyk \& Sposito, 2001):

mol $_{\mathrm{c}} \mathrm{kg}^{-1}-\frac{2 \mathrm{~mol}_{\mathrm{e}}}{\mathrm{mol}_{2}} \times \frac{\text { densidade da solução de } \mathrm{I}_{2} \times \text { molinidade da solução } \mathrm{I}_{2}}{\text { Massa de AH }}$

Determinou-se o ponto de inflexão de cada curva de titulação redox, obtendo-se a segunda derivada da função usando o software Origin ${ }^{\circledR}$. No ponto de inflexão, a f.e.m.cela foi estimada por interpolação (Struyk \& Sposito, 2001). Adicionalmente, realizaram-se titulações de amostras em branco, sem adição de AH.

\section{Análise dos dados das titulações redox}

Analisaram-se os dados obtidos com a titulação redox dos $\mathrm{AH}$ de acordo com estudo desenvolvido inicialmente por Struyk \& Sposito (2001). Os valores de força eletromotriz (f.e.m.cela), medidos durante as titulações redox, foram relacionados com o potencial formal do eletrodo $\left({ }^{\mathrm{F}} \mathrm{E}_{\mathrm{H}}\right)$, de acordo com a equação:

$$
\text { f.e. } \mathrm{m}_{\text {cela }}=\mathrm{F}_{\mathrm{H}}\left(\mathrm{HA}_{\mathrm{Ox}}, \mathrm{HA}_{\mathrm{Red}}\right)-\mathrm{E}_{\mathrm{H}}^{\circ}(\mathrm{AgCl}, \mathrm{Ag})(5)
$$

em que $\mathrm{E}_{\mathrm{H}}{ }^{\circ}(\mathrm{AgCl}, \mathrm{Ag})=222 \mathrm{mV}$ é o potencial-padrão do eletrodo de referência $\left(\mathrm{AgCl}, \mathrm{Ag}\right.$ a $25^{\circ} \mathrm{C}$ ) relativo ao eletrodo-padrão de hidrogênio e ${ }^{\mathrm{F}} \mathrm{E}_{\mathrm{H}}\left(\mathrm{HA}_{\mathrm{ox}}, \mathrm{HA}_{\text {red }}\right)$ é o potencial formal de eletrodo da meia reação $\mathrm{AH}_{\mathrm{Ox}}$, $\mathrm{AH}_{\text {Red }}$, que correspondem, respectivamente, às formas oxidada e reduzida de $\mathrm{AH}$, de acordo com a reação de redução global:

$$
\mathrm{AH}_{\mathrm{Ox}}+\mathrm{ne}^{-}+\mathrm{qH}^{+}=\mathrm{AH}_{\text {Red }}
$$

No ponto de inflexão (Stumm \& Morgan, 1996; Struyk \& Sposito, 2001):

$$
\begin{gathered}
\text { f.e. } \mathrm{m}_{\text {cela }}={ }^{\mathrm{F}} \mathrm{E}_{{ }_{\mathrm{H}}^{\circ}}^{\circ}\left(\mathrm{AH}_{\mathrm{Ox}}, \mathrm{AH}_{\mathrm{Red}}\right)-(\mathrm{q} / \mathrm{n}) 0,05916 \mathrm{pH} \\
-\mathrm{E}_{\mathrm{H}}^{\circ}(\mathrm{AgCl}, \mathrm{Ag})
\end{gathered}
$$

na qual ${ }^{\mathrm{F}} \mathrm{E}_{\mathrm{H}}^{\mathrm{o}}\left(\mathrm{AH}_{\mathrm{ox}}, \mathrm{AH}_{\mathrm{red}}\right)$ indica o potencial formalpadrão do eletrodo de $\mathrm{AH}$.

A relação q/n indica o número de mols de prótons (q) por mols de elétrons (n) transferidos durante a oxidação de 1 mol de AH. Foi calculada utilizando-se os valores da f.e.m. cela, obtidos para dois valores de pH $(5,0$ e 7,0), por meio da equação: 


$$
-\frac{\mathrm{q}}{\mathrm{n}}=\frac{\frac{\Delta \text { f.e.m. }}{0,05916}}{\Delta \mathrm{pH}}
$$

em que $\Delta$ f.e.m. é a variação da f.e.m.cela por unidade $\log$ de variação do $\mathrm{pH}(\Delta \mathrm{pH})$ no ponto de inflexão da titulação.

De posse dos valores da relação q/n e da f.e.m.cela no ponto de inflexão, calculou-se o ${ }^{\mathrm{F}} \mathrm{E}_{\mathrm{H}}^{\mathrm{o}}$ para a meia reação redox $\left(\mathrm{AH}_{\mathrm{Ox}}, \mathrm{AH}_{\mathrm{Red}}\right)$, usando-se a equação 7 .

$\mathrm{O}{ }^{\mathrm{F}} \mathrm{E}_{\mathrm{H}}{ }^{\mathrm{o}}$ de cada amostra de $\mathrm{AH}$ foi convertido na constante de equilíbrio condicional, usando-se a seguinte relação (Stumm \& Morgan, 1996):

$$
\log \mathrm{K}_{\mathrm{c}}=\frac{{ }^{\mathrm{F}} \mathrm{E}_{\mathrm{H}}^{\mathrm{O}}}{0,05916}
$$

na qual $\mathrm{K}_{\mathrm{c}}$ é a constante de equilíbrio condicional, a $298 \mathrm{~K}$, correspondendo à reação de redução genérica para um elétron transferido (Struyk \& Sposito, 2001):

$$
\mathrm{m} \mathrm{Ox}+(\mathrm{q} / \mathrm{n}) \mathrm{H}^{+}+\mathrm{e}^{-}=\mathrm{pRed}
$$

$\mathrm{O}$ pe para o par redox $\mathrm{AH}_{\mathrm{Ox}}, \mathrm{AH}_{\mathrm{Red}}$ foi obtido conforme a equação (Stumm \& Morgan, 1996):

$$
\text { pe }=\log K_{c}-(q / n) p H
$$

\section{Determinação dos grupos funcionais}

A determinação dos grupos funcionais ácidos dos AH foi realizada de acordo com Schnitzer \& Gupta (1965). Determinou-se a acidez total e a carboxílica experimentalmente e, pela sua diferença, a acidez fenólica.

Para determinação da acidez total foram adicionados, em um erlenmeyer de $125 \mathrm{~mL}, 100 \mathrm{mg}$ de $\mathrm{AH}$ e $20 \mathrm{~mL}$ de $\mathrm{Ba}(\mathrm{OH})_{2}$ 0,125 mol L-1 . Simultaneamente, foi preparada uma amostra em branco. O ar do frasco foi trocado por $\mathrm{N}_{2}$ através do borbulhamento por cinco minutos, e o sistema foi agitado por $24 \mathrm{~h}$ à temperatura ambiente $\left(25^{\circ} \mathrm{C}\right)$. A seguir, a suspensão foi filtrada e o resíduo lavado com água destilada livre de $\mathrm{CO}_{2}$. (água fervida e resfriada para $25^{\circ} \mathrm{C}$ ). O excesso de $\mathrm{Ba}(\mathrm{OH})_{2}$ da suspensão foi titulado potenciometricamente com $\mathrm{HCl}$ 0,5 mol L-1, até $\mathrm{pH} 8,4$.

A extração da acidez carboxílica (teor de grupos $\mathrm{COOH}$ ) foi realizada com acetato de cálcio e determinada por titulação com hidróxido de sódio. Em um erlenmeyer de $125 \mathrm{~mL}$ foram adicionados $100 \mathrm{mg}$ de $\mathrm{AH}, 10 \mathrm{~mL}$ de $\mathrm{Ca}(\mathrm{OAc})_{2} 1,0 \mathrm{~mol} \mathrm{~L}^{-1}$ e $40 \mathrm{~mL}$ de água destilada livre de $\mathrm{CO}_{2}$. Simultaneamente, foi preparado o teste em branco. Sob atmosfera de $\mathrm{N}_{2}$, o sistema foi agitado por $24 \mathrm{~h}$ à temperatura ambiente. A seguir, a suspensão foi filtrada e o resíduo lavado com água destilada livre de $\mathrm{CO}_{2}$. O ácido acético produzido pela reação do acetato com o $\mathrm{H}^{+}$do grupo carboxílico foi titulado com $\mathrm{NaOH} 0,1 \mathrm{~mol} \mathrm{~L}^{-1}$, previamente padronizado com biftalato de potássio, até $\mathrm{pH} 9,8$.

A concentração de grupos quinonas nos $\mathrm{AH}$ foi estimada pelo método da redução a hidroquinona, usando-se o cloreto de estanho (II) em meio alcalino como redutor, conforme Schnitzer \& Riffaldi (1972). Adicionaram-se ao frasco $20 \mathrm{mg}$ de $\mathrm{AH}, 0,2 \mathrm{~mL}$ de etanol e $10 \mathrm{~mL}$ de $\mathrm{NaOH} 0,1 \mathrm{~mol} \mathrm{~L}^{-1}$ sob agitação constante até a solubilização. Em seguida, foram adicionados $20 \mathrm{~mL}$ de $\mathrm{NaOH} 2,5 \mathrm{~mol} \mathrm{~L}^{-1}$ e $10 \mathrm{~mL}$ de $\mathrm{SnCl}_{2} 6 \mathrm{H}_{2} \mathrm{O} 0,05 \mathrm{~mol} \mathrm{~L}^{-1}$ e o frasco foi fechado. Procedeu-se à reação por uma hora sob agitação constante e sob atmosfera inerte de $\mathrm{N}_{2}$. O excesso do redutor $\left(\mathrm{Sn}^{2+}\right)$ foi titulado potenciometricamente, usando-se o $\mathrm{Cr}_{2} \mathrm{O}_{7}{ }^{2-}$ como oxidante, a partir da solução titulante $\mathrm{K}_{2} \mathrm{Cr}_{2} \mathrm{O}_{7} 0,05 \mathrm{~mol} \mathrm{~L}^{-1}$.

Os espectros de RPE foram obtidos à temperatura ambiente, usando um espectrômetro Bruker EMX 9 $\mathrm{GHz}$, conforme Martin-Neto et al. (1994a, b). Para obtenção das áreas dos sinais foi utilizada a aproximação I x $\left(\Delta \mathrm{H}_{\mathrm{PP}}\right)^{2}$ (Poole, 1967), em que I é a intensidade do sinal e $\Delta \mathrm{H}_{\mathrm{PP}}$ é a largura deste, tomada de pico a pico. Na determinação da CRLS utilizou-se o método do padrão secundário com um cristal de rubi e o padrão strong pitch da Bruker com concentração de radicais livres (spins $\mathrm{g}^{-1}$ ) conhecida. As condições experimentais foram: potência das microondas de 0,1 mW, determinada por saturação de potência; freqüência de modulação de $100 \mathrm{kHz}$; amplitude de modulação de 0,1 mT; constante de tempo de 2,56 ms; e tempo de conversão de 10,24 ms. As análises foram realizadas com, no mínimo, oito varreduras por determinação.

\section{Resíduos de Fe}

A presença de resíduos de Fe nos $\mathrm{AH}$ foi detectada usando-se energia dispersiva de raios X acoplado a um microscópio eletrônico de varredura (MEV-ESEM 962Zeiss). Obtiveram-se os seguintes espectros: geral (presença do elemento) e pontual (localização do elemento) do campo analisado (Vidal-Torrado et al., 2006).

\section{Análises estatísticas}

Estimaram-se o erro-padrão da média para cada variável experimental e a correlação linear de Pearson entre a capacidade de oxidação e a concentração de grupos funcionais dos AH. As estimativas dos coeficientes de correlação foram submetidas ao teste F a 1 e $5 \%$ (Steel \& Torrie, 1960).

\section{RESULTADOS E DISCUSSÃO}

\section{Potencial do eletrodo}

As curvas de titulação potenciométrica dos $\mathrm{AH}$ foram semelhantes às obtidas por Struyk \& Sposito (2001), usando três amostras-padrão de AH da IHSS. Um exemplo de curva de titulação redox iodimétrica encontra-se na figura 2. 


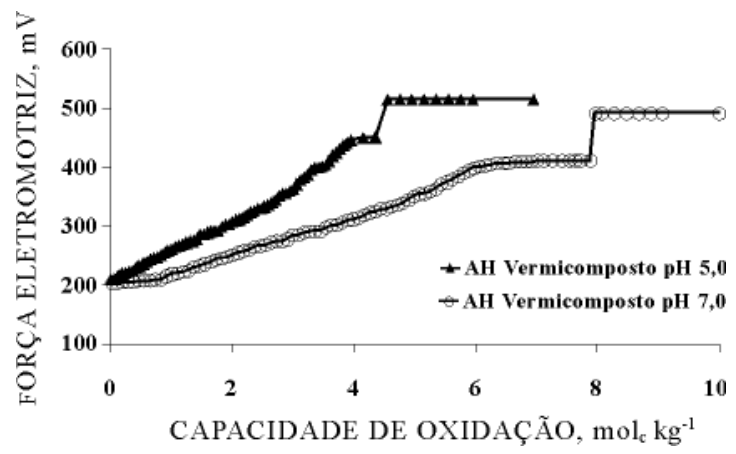

Figura 2. Titulações redox de $6,25 \mathrm{mg}$ de ácido húmico (AH), suspenso em $\mathrm{KCl} 0,05 \mathrm{~mol} \mathrm{~L}^{-1} \mathrm{e}$ solução-tampão fosfato $0,05 \mathrm{~mol} \mathrm{~L}^{-1}$, a pH 5,00 e 7,00 .

Foi possível observar um nítido ponto de inflexão nessas curvas de titulação, que apresentaram a variação característica da f.e.m.cela com a depleção do redutor e adição de oxidante, e, também, uma região com pequena variação da f.e.m.cela. Assumiu-se a inflexão da curva como o ponto final da titulação potenciométrica. Segundo Struyk \& Sposito (2001), a pequena variação da f.e.m.cela está associada à presença de resíduos de $\mathrm{Fe}$ (II) nos $\mathrm{AH}$, não removidos mesmo após o processo de purificação recomendado pela IHSS.

Os dados das titulações redox dos AH revelaram diminuição média de $40 \mathrm{mV}$ na f.e.m. cela com o aumento do $\mathrm{pH}$ de 5,0 para 7,0 (Quadro 1). Essa tendência foi observada por Struyk \& Sposito (2001) e por Matthiessen (1995). A equação 7, apresentada anteriormente, indica que a f.e. $m_{\text {cela }}$ varia inversamente com o $\mathrm{pH}$ do sistema redox. Todos os $\mathrm{AH}$ isolados no presente trabalho transferiram ao $\mathrm{I}_{2}(\mathrm{aq})$, na reação de oxidação, aproximadamente um próton para cada três elétrons, ou seja, a relação $q / n$ foi igual a 0,33. Esse valor médio também foi observado por Struyk \& Sposito (2001) para amostras-padrão de AH da IHSS.

Considerando a reação redox entre $\mathrm{AH}_{\text {Red }}$ e $\mathrm{I}_{2}$, com a relação q/n de aproximadamente 0,33 (1/3 de $\mathrm{H}^{+}$por $\mathrm{e}^{-}$transferido), os resultados permitem supor que o par redox $\mathrm{AH}_{\mathrm{Ox}}, \mathrm{AH}_{\text {Red }}$ resulte na reação de redução genérica dependente do $\mathrm{pH}$ :

$$
\mathrm{AH}_{\mathrm{Ox}}+\mathrm{e}^{-}+1 / 3 \mathrm{H}^{+}=\mathrm{AH}_{\mathrm{Red}}
$$

No presente estudo, os valores de ${ }^{\mathrm{F}} \mathrm{E}_{\mathrm{H}}{ }^{\circ}$ para a semireação de redução dos $\mathrm{AH}$ isolados dos adubos orgânicos foram semelhantes e, em média, iguais a 0,782 V. Struyk \& Sposito (2001), usando três amostras de $\mathrm{AH}$ obtidas de solo e de sedimentos, observaram valores de ${ }^{\mathrm{F}} \mathrm{E}_{\mathrm{H}}{ }^{\mathrm{o}}$ próximos a $0,780 \mathrm{~V}$. Ambos os trabalhos usaram o $\mathrm{I}_{2}$ como oxidante. Os

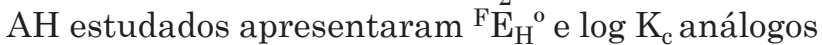
aos de compostos o-quinona (Helburn \& MacCarthy, 1994). No entanto, diferenciam-se pela mais ampla relação $q / n$ verificada nos grupos o-quinona $(1,00) \mathrm{em}$ relação aos $\mathrm{AH}(0,33)$.

A comparação entre os valores das propriedades redox de alguns pares comuns nos sistemas naturais (Quadro 2) permitiu alocar o par redox $\mathrm{AH}_{\mathrm{Ox}}, \mathrm{AH}_{\mathrm{Red}}$, com a média de $\mathrm{F}_{\mathrm{H}}{ }^{\circ} \sim 0,782 \mathrm{~V}$, na "seqüência redox" indicada por Stumm \& Morgan (1996). Os resultados apresentados confirmaram que os $\mathrm{AH}$ podem reduzir $\mathrm{Hg}(\mathrm{II})$ e Mn(IV), conforme observaram, respectivamente, Alberts et al. (1974) e Sunda \& Kieber (1994), incubando tais íons metálicos com bactérias redutoras e $\mathrm{AH}$.

Introduzindo os dados médios dos $\mathrm{AH}$ estudados no diagrama pe vs $\mathrm{pH}$ adaptado de Bartlett \& James (1993), contendo os dados das amostras-padrão de AH

Quadro 1. Capacidade de oxidação $(\mathrm{COx})$ e potencial formal-padrão do eletrodo $\left({ }^{\mathrm{F}} \mathrm{E}_{\mathrm{H}}^{\mathrm{o}}\right)$ de ácidos húmicos

\begin{tabular}{|c|c|c|c|c|c|c|c|c|}
\hline \multirow{2}{*}{ Ácido húmico } & \multicolumn{2}{|c|}{$\mathrm{COx}$} & \multicolumn{2}{|c|}{ f.e.m.cela } & \multirow[b]{2}{*}{$\mathbf{q} / \mathbf{n}$} & \multirow[b]{2}{*}{$\mathrm{F}^{\mathrm{o}}{ }_{\mathrm{H}}$} & \multirow[b]{2}{*}{$\log \mathrm{K}_{\mathrm{c}}$} & \multirow[b]{2}{*}{$\mathbf{p e}_{\mathrm{c}}$} \\
\hline & pH 5 & pH 7 & pH 5 & pH 7 & & & & \\
\hline & \multicolumn{2}{|c|}{ mol $_{\mathrm{c}} \mathrm{kg}^{-1}$} & \multicolumn{2}{|c|}{$\mathrm{mV}$} & \multicolumn{3}{|c|}{$\mathrm{V}$} & \\
\hline Vermicomposto & $4,39 \pm 0,05$ & $7,89 \pm 0,09$ & $451 \pm 3$ & $411 \pm 5$ & 0,34 & 0,773 & 13,1 & 10,7 \\
\hline Torta de Filtro & $4,20 \pm 0,04$ & $6,23 \pm 0,06$ & $459 \pm 5$ & $420 \pm 3$ & 0,33 & 0,779 & 13,2 & 10,9 \\
\hline Esterco de Curral & $4,25 \pm 0,07$ & $7,35 \pm 0,09$ & $460 \pm 4$ & $421 \pm 5$ & 0,33 & 0,780 & 13,2 & 10,9 \\
\hline Esterco de Aviário & $4,35 \pm 0,05$ & $7,64 \pm 0,08$ & $465 \pm 2$ & $425 \pm 4$ & 0,34 & 0,787 & 13,3 & 10,9 \\
\hline Curral + Aviário & $4,28 \pm 0,06$ & $6,99 \pm 0,09$ & $455 \pm 2$ & $415 \pm 6$ & 0,33 & 0,777 & 13,1 & 10,8 \\
\hline Lixo Urbano & $3,88 \pm 0,05$ & $5,35 \pm 0,05$ & $472 \pm 3$ & $432 \pm 4$ & 0,34 & 0,794 & 13,4 & 11,0 \\
\hline
\end{tabular}

As médias dos valores determinados experimentalmente para a COx e a f.e.m.cela estão acompanhadas pelo seu erro-padrão. As demais variáveis foram calculadas como descrito na seção Material e Métodos. 
Quadro 2. Propriedades redox de alguns pares comuns em sistemas naturais

\begin{tabular}{|c|c|c|c|c|}
\hline Pares redox & ${ }^{\mathrm{F}} \mathbf{E}_{\mathrm{H}}{ }^{\mathrm{o}}$ & $\log K_{\mathrm{c}}$ & pe $_{c}^{(1)}(\mathrm{pH} 5)$ & $\mathbf{p e}_{\mathrm{c}}(\mathbf{p H} 7)$ \\
\hline & $\mathrm{V}$ & & & \\
\hline $1 / 2 \mathrm{MnO}_{2}(\mathrm{~s})+2 \mathrm{H}^{+}+\mathrm{e}^{-}=1 / 2 \mathrm{Mn}^{2+}(\mathrm{aq})+\mathrm{H}_{2} \mathrm{O}$ & 0,76 & 12,8 & 12,8 & 8,8 \\
\hline $\mathrm{Fe}(\mathrm{OH})_{2}+(\mathrm{aq})+\mathrm{e}^{-}+\mathrm{H}^{+}=\mathrm{Fe}^{2+}(\mathrm{aq})+\mathrm{H}_{2} \mathrm{O}$ & 0,90 & 15,2 & 10,2 & 8,2 \\
\hline $1 / 2 \mathrm{Hg}^{2+}(\mathrm{aq})+\mathrm{e}^{-}=\mathrm{Hg}^{\circ}(\mathrm{g})$ & 0,85 & 14,4 & 14,4 & 14,4 \\
\hline $\mathrm{AH}_{\mathrm{ox}}+1 / 3 \mathrm{H}^{+}+\mathrm{e}^{-}=\mathrm{AH}_{\mathrm{red}}$ & 0,78 & 13,2 & 11,5 & 10,9 \\
\hline $\mathrm{FeOOH}(\mathrm{s})+\mathrm{e}^{-}+3 \mathrm{H}^{+}=\mathrm{Fe}^{2+}(\mathrm{aq})+\mathrm{H}_{2} \mathrm{O}$ & 0,77 & 13,0 & 2,0 & $-4,0$ \\
\hline $\mathrm{Fe}^{3+}(\mathrm{aq})+\mathrm{e}^{-}=\mathrm{Fe}^{2+}(\mathrm{aq})$ & 0,77 & 13,0 & 13,0 & 13,0 \\
\hline
\end{tabular}

(1) Os valores de pe foram calculados usando-se os valores de $\log \mathrm{K}_{\mathrm{c}}$, concentração de redutores e oxidantes $=10^{-4} \mathrm{~mol} \mathrm{~L}^{-1}$ de espécies solúveis, atividade das fases sólidas iguais a 1 e $\mathrm{P}_{\mathrm{O} 2}=0,21 \mathrm{~atm}$. Os valores em negrito são as médias dos dados obtidos com os ácidos húmicos (AH) isolados dos adubos orgânicos.

da IHSS (Struyk \& Sposito, 2001), foi possível obter informações sobre a atividade das suas espécies $\left(\mathrm{AH}_{\mathrm{Ox}}\right.$, Red ) nos sistemas naturais (Figura 3). De acordo com o diagrama pe vs $\mathrm{pH}$, assumiu-se que todos os $\mathrm{AH}$ estudados predominavam na forma $\mathrm{AH}_{\text {Red }}$, podendo atuar como doadores de elétrons nas reações redox ocorrentes nos sistemas naturais. Assim, o $\mathrm{Fe}^{3+}$ solúvel seria uma espécie termodinamicamente favorável à redução por $\mathrm{AH}$, embora $\mathrm{Fe}(\mathrm{OH})_{2}{ }^{+}$possa ser a espécie predominante em solução a $\mathrm{pH}$ entre 5 e 7 , respectivamente. Como o par redox $\mathrm{Fe}^{2+}, \mathrm{FeOOH}$ está abaixo do par redox $\mathrm{AH}_{\mathrm{Ox}}$, Red entre os valores de $\mathrm{pH} 5$ e $7, \mathrm{FeOOH}$ e $\mathrm{AH}_{\mathrm{Red}}$ poderiam predominar termodinamicamente. Segundo Lovley et al. (1996, 1998) e Scott et al. (1998), os AH poderiam mediar a redução microbiana de $\mathrm{Fe}(\mathrm{III})$ a partir da fase sólida. Se bactérias redutoras podem acessar $\mathrm{Fe}$ (III) em óxidos como, por exemplo, os da espécie FeOOH (Lovley et al., 1996, 1998, 2000; Scott et al., 1998), é possível que após a redução microbiana os $\mathrm{AH}$ apresentem potencial abaixo do determinado no presente estudo (na ausência de microrganismos).

\section{Capacidade de oxidação}

As titulações realizadas a $\mathrm{pH} 7,0$ resultaram em incrementos médios na $\mathrm{COx}$ dos $\mathrm{AH}$ de $64 \%$ sobre os valores a $\mathrm{pH} 5,0$, indicando que tal propriedade foi dependente do $\mathrm{pH}$. Esses resultados são corroborados pelos estudos de Szilâgyi (1973) e de Stuyk \& Sposito (2001). O aumento da COx com o $\mathrm{pH}$ pode ser entendido de acordo com a equação 6 .

A origem dos adubos orgânicos também modificou a $\mathrm{COx}$ dos $\mathrm{AH}$, que variou entre 3,88 e $4,39 \mathrm{~mol}_{\mathrm{c}} \mathrm{kg}^{-1}$ nas titulações redox a pH 5,0 e de 5,35 a 7,89 mol $_{\mathrm{c}} \mathrm{kg}^{-1}$ a pH 7,0. Struyk \& Sposito (2001), em três amostraspadrão da IHSS, encontraram COx variando de 1,09 a $6,5 \mathrm{~mol}_{\mathrm{c}} \mathrm{kg}^{-1} \mathrm{e} 3,30$ a $11,50 \mathrm{~mol}_{\mathrm{c}} \mathrm{kg}^{-1}$, nas titulações a pH 5,0 e 7,0, respectivamente. Matthiessen (1995), em amostras de $\mathrm{AH}$ sintético e usando o $\mathrm{I}_{2}$ como

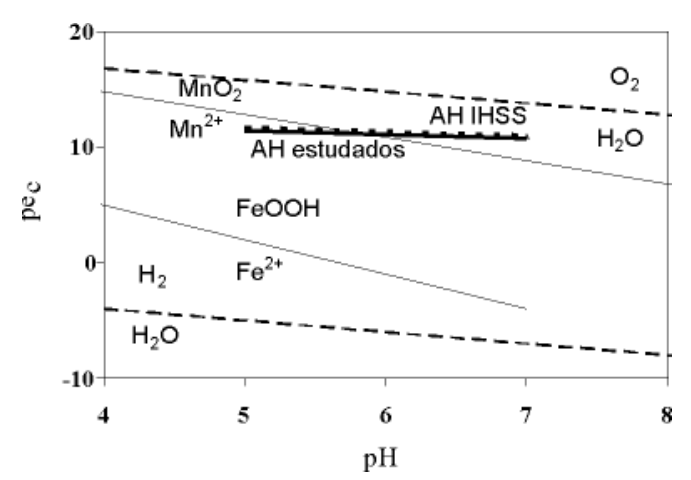

Figura 3. Diagrama pe vs pH, mostrando pares redox comuns em sistemas naturais e $\mathrm{AH}$ isolados de adubos orgânicos e de amostras-padrão da IHSS (Struyk \& Sposito, 2001). Adaptado de Bartlett \& James (1993).

oxidante, encontrou valores médios de $7,5 \mathrm{~mol}_{\mathrm{c}} \mathrm{kg}^{-1} \mathrm{a}$ pH 5,0 e de 10,5 mol $_{\mathrm{c}} \mathrm{kg}^{-1}$ a pH 7,0.

Os valores da $\mathrm{COx}$ dos $\mathrm{AH}$ decresceram na ordem: vermicomposto $>$ estercos de curral e de aviário $>$ torta de filtro > lixo urbano. Essa diminuição da $\mathrm{COx}$ foi acompanhada pelo decréscimo das concentrações de grupos funcionais (Quadro 3).

\section{Grupos funcionais}

A correlação entre a COx e a concentração de grupos funcionais semiquinonas $(0,97$ a pH 5 e 0,99 a pH 7), quinonas $(0,96$ a pH 5 e 0,98 a pH 7) e fenólicos $(0,92$ a $\mathrm{pH} 5$ e $0,99 \mathrm{a} \mathrm{pH}$ 7) dos $\mathrm{AH}$ foi positiva e significativa $(\mathrm{P} \leq 0,01)$. Não se verificou, entretanto, correlação com a concentração de grupos carboxílicos.

Scott et al. (1998) obtiveram correlações positivas e significativas entre a $\mathrm{COx}$ determinada na presença de microrganismos e a concentração de quinonas de AH. Helburn \& MacCarthy (1994) atribuíram os valores obtidos de $\mathrm{COx}$ nas titulações redox de $\mathrm{AH}$ 
Quadro 3. Concentração de grupos funcionais em ácidos húmicos isolados de adubos orgânicos

\begin{tabular}{lcccc}
\hline \multirow{2}{*}{ Ácido húmico } & \multicolumn{3}{c}{ Grupos funcionais $^{(1)}$} \\
\cline { 2 - 4 } & Carboxílicos & Fenólicos & Quinonas & Semiquinonas \\
\cline { 2 - 4 } & & & & $\left(\times 10^{17}\right) \mathrm{spins}^{-1}$ \\
Vermicomposto & $304 \pm 2,53$ & $385 \pm 2,87$ & $105 \pm 0,96$ & $3,13 \pm 0,09$ \\
Torta de Filtro & $351 \pm 3,11$ & $294 \pm 1,54$ & $94 \pm 0,84$ & $2,53 \pm 0,06$ \\
Esterco de Curral & $299 \pm 2,39$ & $320 \pm 1,82$ & $99 \pm 0,91$ & $2,89 \pm 0,08$ \\
Esterco de Aviário & $318 \pm 3,08$ & $335 \pm 2,01$ & $104 \pm 0,98$ & $3,01 \pm 0,08$ \\
Curral + Aviário & $302 \pm 2,15$ & $310 \pm 1,77$ & $101 \pm 0,89$ & $2,67 \pm 0,07$ \\
Lixo Urbano & $322 \pm 2,61$ & $98 \pm 0,89$ & $88 \pm 0,78$ & $1,95 \pm 0,06$ \\
\hline
\end{tabular}

(1) As médias experimentais estão acompanhadas pelo seu erro-padrão.

sintéticos à presença de grupos fenólicos, dada a estreita relação com a acidez fenólica dos $\mathrm{AH}$ estudados. Struyk \& Sposito (2001) não estimaram as correlações, mas sugeriram a relação entre a $\mathrm{COx}$ e a concentração de grupos funcionais semiquinonas dos AH. Todos os trabalhos supracitados também relataram a ausência de correlação entre a concentração de grupos carboxílicos e a COx dos AH.

Struyk \& Sposito (2001) estimaram as contribuições de grupos funcionais semiquinonas no poder redutor dos AH e concluíram que os valores da $\mathrm{COx}$ dos três $\mathrm{AH}$ estudados não foram suficientemente explicados pela CRLS. Seguindo a estimativa apresentada por Struyk \& Sposito (2001), as titulações redox dos AH isolados dos adubos orgânicos também resultaram em valores de $\mathrm{COx}$ superiores à potencial contribuição da CRLS, determinada por RPE. Para exemplificar, usou-se a CRLS do AH isolado do vermicomposto, igual a 4,64 $10^{17}$ spins g-1. Esses radicais livres contribuiriam com uma fração de aproximadamente $7,7110^{-4}$ $\mathrm{mol}_{\mathrm{c}}(\mathrm{kg} \mathrm{AH})^{-1}$, aquém da $\mathrm{COx}$ determinada, igual a $7,89 \mathrm{~mol}_{\mathrm{c}} \mathrm{kg}^{-1}$ Dados indiretos sobre o poder redutor dos $\mathrm{AH}$, obtidos a partir da medição das espécies reduzidas de metais como o Fe (Wilson \& Weber, 1979; Scott et al., 1998), após incubação com microrganismos e AH, indicaram que a CRLS poderia ser responsável por no máximo $10 \%$ da quantidade de elétrons transferidos. Os resultados permitem inferir as contribuições de grupos quinonas e fenólicos dos $\mathrm{AH}$ na sua capacidade de transferência de elétrons.

Dois mecanismos, anteriormente usados na interpretação da capacidade redox das $\mathrm{SH}$, podem, concomitantemente, explicar a alta correlação $(0,97$ a pH 5 e 0,99 a pH $7, \mathrm{P} \leq 0,01$ ) obtida entre a COx e a CRLS dos AH, considerando também as relações da COx com grupos quinonas e fenólicos: (a) a redução de grupos quinonas e fenólicos a semiquinonas proposta por Scott et al. (1998); e (b) a oxidação iodimétrica do complexo Fe-grupos fenólicos dos AH, gerando radicais livres semiquinonas - propostos por Struyk \& Sposito (2001). Adicionalmente, o sinal de RPE desaparece com as reações citadas por Scott et al. (1998) para a formação das espécies hidroquinonas e benzoquinonas a partir de semiquinonas, durante a titulação redox. Apesar de não serem determinados por RPE, esses radicais podem ter sido oxidados pelo $\mathrm{I}_{2}$ nas condições especificadas no presente estudo, contribuindo para o aumento dos valores da $\mathrm{COx}$ dos $\mathrm{AH}$.

\section{Derivados metilados e acetilados}

As curvas de titulação potenciométrica foram drasticamente modificadas pela metilação e a acetilação dos AH (Figura 4). Esses processos reduziram em $94 \%$ a $\mathrm{COx}$ dos $\mathrm{AH}$, permitindo mostrar a importante contribuição dos grupos fenólicos e quinonas livres na transferência de elétrons nos $\mathrm{AH}$.

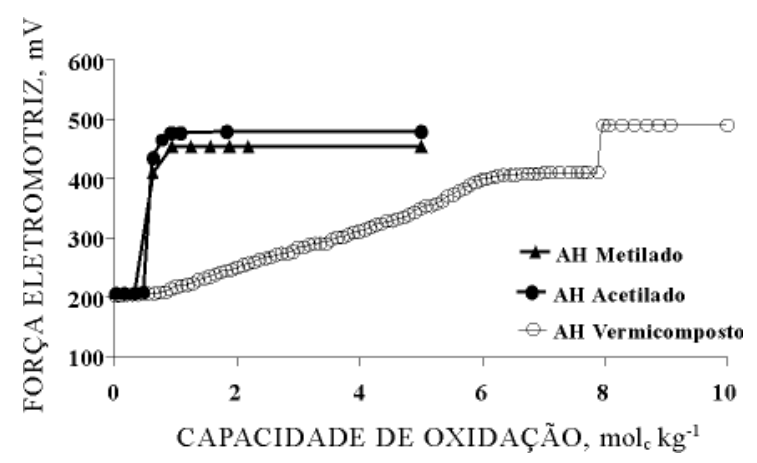

Figura 4. Titulações redox de $6,25 \mathrm{mg}$ de ácido húmico e de 6,25 mg de seus derivados obtidos por metilação e acetilação, suspensos em $125 \mathrm{~mL}$ de $\mathrm{KCl} 0,05 \mathrm{~mol} \mathrm{~L}^{-1}$ e solução-tampão fosfato $0,05 \mathrm{~mol} \mathrm{~L}^{-1}$, a pH 7,00. 
O monitoramento das reações de metilação e de acetilação dos $\mathrm{AH}$, realizado por meio da espectroscopia na região do IV (Figura 5), indicou que a metilação dos AH converteu grupos oxigenados em ésteres. A acetilação "bloqueou" os grupos fenólicos e quinonas livres. Os espectros de IV-TF mostraram que a intensidade de absorção em $1.735-1.742 \mathrm{~cm}^{-1}$ aumentou consideravelmente com a metilação dos $\mathrm{AH}$. A absorção, devido ao estiramento C-H (2.927$\left.2.942 \mathrm{~cm}^{-1}\right)$, foi também aumentada pela metilação e acetilação.

Os resultados das titulações redox dos derivados metilados e acetilados dos $\mathrm{AH}$ indicaram que, em média, $6 \%$ da $\mathrm{COx}$ poderia ser atribuída, por exemplo,
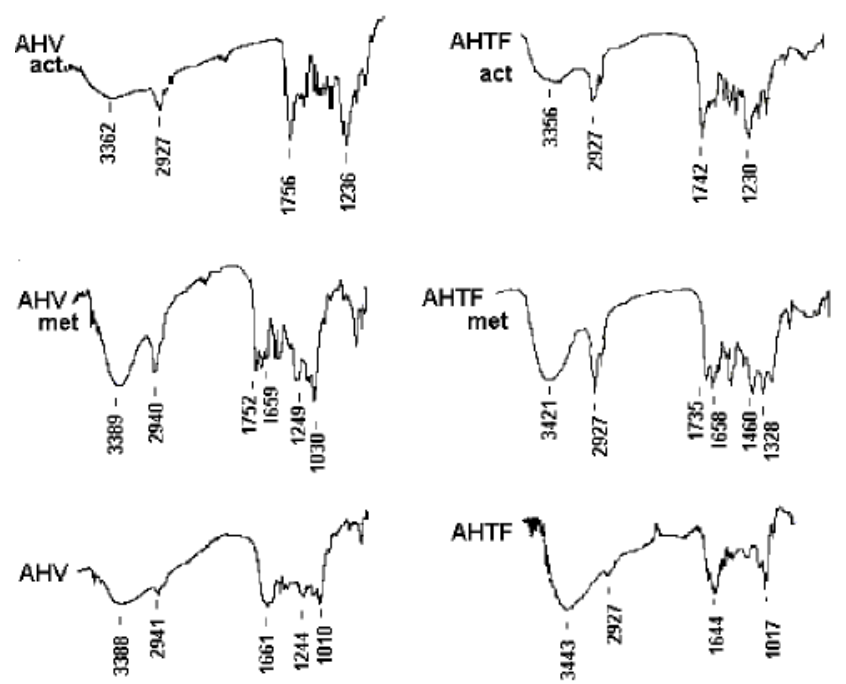

Figura 5. Espectros de infravermelho com transformada de Fourier e atribuições das regiões de absorção (número de onda, $\mathrm{cm}^{-1}$ ) para os ácidos húmicos isolados de vermicomposto (AHV) e seus derivados metilados (AHVmet) e acetilados (AHVact) e de torta de filtro (AHTF) e seus derivados metilados (AHTFmet) e acetilados (AHTFact). ao mecanismo do Fe(II) ligado aos AH. Struyk \& Sposito (2001), com base na concentração de cinzas de amostras-padrão de AH da IHSS, estimaram a participação do Fe na $\mathrm{COx}$ dos $\mathrm{AH}$. Os autores consideraram um teor de cinzas nos $\mathrm{AH}$ de $3,10 \%$. Assim, usando os $6,25 \mathrm{mg}$ de $\mathrm{AH}$ das titulações e assumindo que a espécie $\mathrm{Fe}_{2} \mathrm{O}_{3}$ determinava toda a concentração de cinzas, indicaram que aproximadamente $0,14 \mathrm{~mol} \mathrm{~kg}^{-1}$ de Fe estava presente nos AH. Cada mol de $\mathrm{Fe}(\mathrm{II})$ pode transferir $1 \mathrm{~mol}_{\mathrm{c}}$ para $\mathrm{I}_{2}$. Sendo a média da COx das amostras-padrão de AH estudadas por Struyk \& Sposito (2001) igual a $6,91 \mathrm{~mol}_{\mathrm{c}} \mathrm{kg}^{-1}$ a $\mathrm{pH} 7,0$, a porcentagem indicada de Fe resultou na $\mathrm{COx}$ de $0,41 \mathrm{~mol}_{\mathrm{c}} \mathrm{kg}^{-1}$.

Nos AH dos adubos orgânicos foi detectada a presença de resíduos de Fe, por meio da microscopia eletrônica de varredura (Figura 6). De posse dos resultados da microscopia analítica, indicando a presença de $\mathrm{Fe}$ nos $\mathrm{AH}$, usou-se o procedimento apresentado por Struyk \& Sposito (2001) para estimar a participação do Fe na capacidade de oxidação dos AH isolados dos adubos orgânicos. As amostras estudadas apresentaram, em média, 3,6 \% de cinzas, indicando que esses $\mathrm{AH}$ poderiam apresentar $\mathrm{COx}$ proveniente do $\mathrm{Fe}(\mathrm{II})$, com magnitude de aproximadamente $0,17 \mathrm{~mol}_{\mathrm{c}} \mathrm{kg}^{-1}$. Os teores de cinza dos AH variaram de 3,45 a 3,82\% e correlacionaramse com a COx em ambos os valores de $\mathrm{pH}(\mathrm{r}=0,89 \mathrm{a}$ pH 5 e r $=0,97$ a pH 7, P $\leq 0,01)$. Dessa forma, sendo ambas as estimativas citadas convergentes e provindo de diferentes formas de aproximação, admitiu-se sua semelhança.

Assumindo-se que o ${ }^{\mathrm{F}} \mathrm{E}_{\mathrm{H}}{ }^{\circ}$ do par redox $\mathrm{AH}_{\mathrm{Ox}}, \mathrm{AH}_{\text {Red }}$ defina o seu poder redutor e a COx a quantidade de elétrons transferíveis, a soma das contribuições dos grupos semiquinonas dos AH, já presentes ou alterados durante a titulação, poderia explicar a magnitude dos valores de $\mathrm{COx}$ encontrados. A relação direta entre a COx e a CRLS indica a oxidação desses radicais pelo $\mathrm{I}_{2}$, possibilitando estimar o seu grau de humificação.
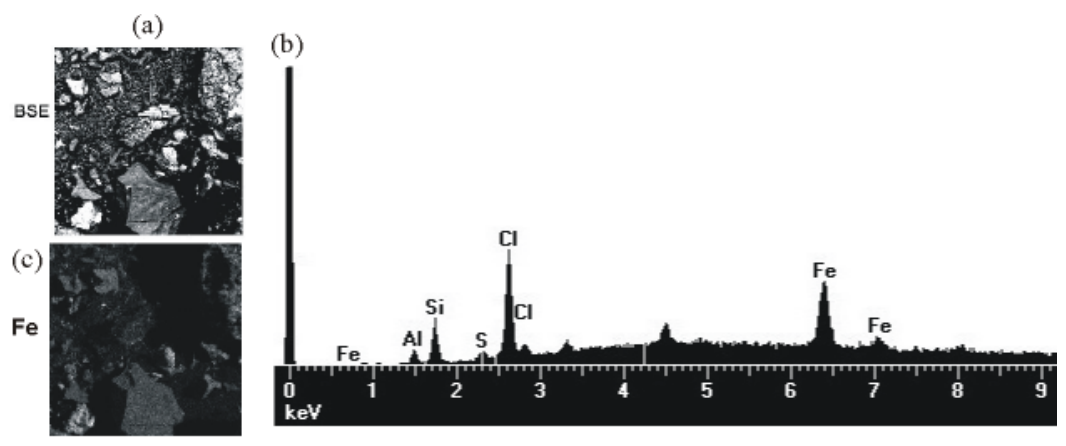

Figura 6. Presença de ferro em ácido húmico (AH) isolado de vermicomposto, obtida por microscopia eletrônica de varredura. (a) imagem em backscattered elétrons (BSE) da amostra de AH; (b) espectro obtido por microscopia analítica, utilizando detector de energia dispersiva de raios $\mathrm{X}$, indicando a presença de ferro no $\mathrm{AH}$; (c) distribuição do ferro na amostra de $\mathrm{AH}$. 


\section{CONCLUSÕES}

1. Os valores do ${ }^{\mathrm{F}} \mathrm{E}_{\mathrm{H}}{ }^{\circ}$ para a semi-reação de redução do par redox $\mathrm{AH}_{\mathrm{Ox}}, \mathrm{AH}_{\text {Red }}$ foram semelhantes entre os AH estudados, sendo, em média, de 0,782 V.

2. A COx dos $\mathrm{AH}$ variou com o $\mathrm{pH}$ do sistema e com o tipo de resíduo orgânico estudado, sendo de 3,88 a $4,39 \mathrm{~mol}_{\mathrm{c}} \mathrm{kg}^{-1}$ a pH 5,0 e de 5,35 a 7,89 mol $_{\mathrm{c}} \mathrm{kg}^{-1} \mathrm{a}$ $\mathrm{pH} 7,0$.

3. As reações de derivação química por metilação e acetilação reduziram drasticamente e de forma semelhante a $\mathrm{COx}$ dos $\mathrm{AH}$.

4. Os AH com maiores teores de grupos fenólicos, quinonas e semiquinonas apresentaram $\mathrm{COx}$ mais elevada.

5. Foi possível predizer a CRLS, bem como o grau de humificação dos AH estudados, a partir da sua COx.

\section{AGRADECIMENTOS}

Aos Drs. Fábio Lopes Olivares (Prof. do Laboratório de Biociências e Biotecnologia-UENF) e Carlos Roberto Matos (Laboratório de Ciências Químicas-UENF), pelas análises microscópicas e reações de metilação, respectivamente. Aos Profs. Gabriel de Araújo Santos (Departamento de Solos-UFRRJ) e Nelson Garcés Pérez (Facultad de Agronomía de la UNAH, Cuba), pelas valiosas sugestões ao trabalho.

\section{LITERATURA CITADA}

ALBERTS, J.J.; SCHINDLER, J.E. \& MILLER, R.W. Elemental mercury evolution mediated by humic acid. Science, 184:895-896, 1974.

BARD, A.; PARSON, R. \& JORDAN, J., eds. Standard potentials in aqueous solution. New York, Marcel Dekker, 1985. 185 p.

BARTLETT, R.J. \& JAMES, B.R. Redox chemistry of soils. Adv. Agron., 50:151-208, 1993.

BENZ, M.; SCHINK, B. \& BRUNE, A. Humic acid reduction by Propionibacterium freudenreichii and outher fermenting bacteria. Appl. Envriron., 64:4507-4512, 1998.

BUDZIAK, C.R.; MAIA, C.M.B.F. \& MANGRICH, A.S. Transformações químicas da matéria orgânica durante a compostagem de resíduos da indústria madeireira. Química Nova, 27:399-403, 2004.

CHEN, Y.; SENESI, N. \& SCHNITZER, M. Information provided on humic substances by $\mathrm{E}_{4} / \mathrm{E}_{6}$ ratio. Soil Sci. Am. J., 41:352-358, 1977.
HELBURN, R.S. \& MACCARTHY, P. Determination of some redox properties of humic acids by alkaline ferricyanide titration. Anal. Chem. Acta, 295:263-272, 1994.

INTERNATIONAL HUMIC SUBSTANCES SOCIETY - IHSS. Products. Disponível em: <http://www.ihss.gatech.edu/ products.html $>$ Acesso em 20 Jul. 2006.

LIGHT, T.S. Standard solution for redox potential measurements. Anal. Chem., 44:1038-1039, 1972.

LOVLEY, D.R.; COATES, J.D.; BLUNT-HARRIS, E.L.; PHILLIPS, E.J.P. \& WOODWARD, J.C. Humic substances as electron acceptors for microbial respiration. Nature, 382:445-448, 1996.

LOVLEY, D.R.; KASHEFI, K.; VARGAS, M.; TOR, J.M. \& BLUNT-HARRIS, E.L. Reduction of humic substances and $\mathrm{Fe}(\mathrm{III})$ by hyperthermophilic microorganisms. Chem. Geol., 169:289-298, 2000.

LOVLEY, D.R.; WOODWARD, J.C.; BLUNT-HARRIS, E.L.; HAYES, L.; PHILLIPS, E.J.P. \& COATES, J.D. Humic substances as mediator for microbially-catalized metal reduction. Acta Hidrochim. Hydrobiol., 26:152-157, 1998.

MARTIN-NETO, L.; ANDRIULO, A.E. \& TRAGHETTA, D. Effects of cultivation on ESR spectra of organic matter from soil size fractions of a mollisol. Soil Sci., 157:365372, 1994a.

MARTIN-NETO, L.; VIEIRA, E.M. \& SPOSITO, G. Mechanism of atrazine sorption by humic acid: a spectroscopic study. Environ. Sci. Technol., 28:1867-1873, 1994b.

MATTHIESSEN, A. Determining the redox capacity of humic substances as a function of $\mathrm{pH}$. Vom Wasser, 84:229-235, 1995.

MILORI, D.M.B.P.; MARTIN NETO, L.; BAYER, C.; MIELNICZUCK, J. \& BAGNATO, V.S. Humification degree of soil humic acids determined by fluorescence spectroscopy. Soil Sci., 167:739-749, 2002.

PÉREZ, M.G.; MARTIN-NETO, L.; SAAB, S.C.; NOVOTNY, E.H.; MILORI, D.M.B.P.; BAGNATO, V.S.; COLNAGO, L.A.; MELO, W.J. \& KNICKER, H. Characterization of humic acids from a Brazilian Oxisol under different tillage systems by EPR, 13C NMR, FTIR and fluorescence spectroscopy. Geoderma, 118:181-190, 2004.

PICCOLO, A. The supramolecular structure of humic substances. Soil Sci., 166:810-832, 2001.

POOLE, C.P. Electron spin resonance: A comprehensive treatise on experimental techniques. New York, WileyInterscience, 1967. 921p.

RIVERO, C.; CHIRENGE, T.; MA, L.Q. \& MARTINEZ, G. Influence of compost on organic soil matter quality under tropical conditions, Geoderma, 123:355-361, 2004.

SACHS, S.; BUBNER, M.; SCHMEIDE, K.; CHOPPIN, G.R.; HEISE, K.H. \& BERNHARD, G. Carbon-13 NMR spectroscopy studies on chemically modified and unmodified synthetic and natural humic acids. Talanta, 57:999-1009, 2002. 
SCHNITZER, M. \& GUPTA, U. C. Determination of acidity in soil organic matter. Soil Sci. Soc. Am. Proc., 29:274-277, 1965.

SCHNITZER, M. \& RIFFALDI, R. The determination of quinone groups in humic substances. Soil Sci. Soc. Am. Proc., 36:772-777, 1972.

SCHWARZENBACH, R.P.; STIERLI, R.; LANZ, K. \& ZEYER, J. Quinone and iron porphyrin mediated reduction of nitroaromatic compounds in homogeneous aqueous solution. Environ. Sci. Tencnol., 24:1566-1574, 1990.

SCOTT, D.T.; MCKNIGHT, D.M.; HARRIS, E.; KOLESAR, S. \& LOVLEY D. Quinone moieties act as electron aceptors in the reducting of humic substances by humics-reducting microorganisms. Environ. Sci. Technol., 32:2984-2989, 1998.

SPOSITO, G. Chemistry of soil. New York, Oxford University Press, 1989. 277p.

STEEL, R.G.D. \& TORRIE, J.H. Principles and procedures of staristics with special referende to the biological sciences. New York, McGraw-Hill Book Company, 1960. 453p.
STEVENSON, F.J. Humus chemistry: Genesis, composition, reactions. 2.ed. New York, John Wiley \& Sons, 1994. 496p.

STRUYK, Z. \& SPOSITO, G. Redox properties of standard humic acids. Geoderma, 102:329-346, 2001.

STUMM, W. \& MORGAN, J.J. Aquatic chemistry: Chemical equilibria and rates in natural waters. New York, WileyInterscience, 1996.

SUNDA, W.G. \& KIEBER, D.J. Oxidation of humic substances by manganese oxides yields low-molecular-weight organic substrates. Nature, 367:62-65, 1994.

SZILÂGUY, M. The redox properties and the determination of the normal potential of peat-water system. Soil Sci., 115:434-437, 1973.

VIDAL-TORRADO, P.; MACIAS, F.; CALVO, R.; CARVALHO, S.G. \& SILVA, A.C. Gênese de solos derivados de rochas ultramáficas serpentinizadas no sudoeste de Minas Gerais. R. Bras. Ci. Solo, 30:553-541, 2006.

WILSON, S.A. \& WEBER, J.H. An EPR study of the reduction of vanadium (V) to vanadium (IV) by fulvic acid. Chem. Geol., 26:1240-1246, 1998. 